\title{
Integrated design of structural and control systems with a homotopy like iterative method
}

\author{
K. HIRAMOTO*† and K. M. GRIGORIADIS \\ $\uparrow$ Department of Mechanical Engineering, Akita University, \\ 1-1 Tegata-gakuen-machi Akita 010-8502, Japan \\ fDepartment of Mechanical Engineering, University of Houston, \\ Houston, TX 77204, USA
}

(Received 15 January 2006; in final form 11 April 2006)

\begin{abstract}
We consider an integrated design problem of structural and control systems. It is well known that even the simplest formulation of this problem results in a kind of a BMI problem. In this paper, homotopy-like iterative design method based on LMIs is proposed to obtain an optimal plant and a controller simultaneously for a full-order output feedback problem. We can deal with a multiobjective problem, e.g., $\mathcal{H}_{2} / \mathcal{H}_{\infty}$ control problem etc. in the proposed design method. We can also optimize structural design parameters appearing non-linearly in the coefficient matrices of the plant state space form using the first order approximation of the non-linear dependence in the proposed algorithm. Several design examples show that the proposed algorithm works quite effectively for various integrated design problems.
\end{abstract}

\section{Introduction}

The objective of control system design is to shape the behaviour of the closed-loop system satisfactory. Generally such optimization has been carried out by synthesizing the optimal controller (in some sense) for a given plant and information about the plant uncertainty. Enormous methodologies have been proposed to obtain the optimal controller for the fixed plant so far and we are able to choose the most appropriate design scheme fitting the objective of the control system designer from the huge catalogue of the design techniques. Especially, the recent development of the LMI approach (Boyd et al. 1994, Scherer et al. 1997, Skelton et al. 1998) has enabled us to design a controller that satisfied various types of closed-loop constraints quite efficiently in the case of a state feedback or fullorder controller design problem.

Meanwhile design parameters in the plant are generally determined with some other specifications, e.g., the strength, the weight and the cost of the structure, etc., before the controller design in most cases. In the above two-step design methodology,

*Corresponding author. Email: hira@ipc.akita-u.ac.jp i.e., the plant is firstly fixed and the controller is obtained for the fixed plant, we do not utilize the full design freedom to obtain the optimal control system. In other words, we could not have used the design freedom of the plant to optimize the performance of the controlled closed-loop system. This limitation of the design freedom may become a serious obstacle when a highly tight specification is imposed on the closed-loop system and should be resolved in some sense for the genuine optimal control systems design.

The concept of integrated design of structural and control systems (shortly, we may use the term integrated design in the rest of the paper), is a prescription for the above problem in the general and current framework of control system design. In the integrated design scheme design parameters both in the plant and the controller are dealt with equally and optimized simultaneously. Therefore, we can expect to obtain a better closed-loop performance than that of the conventional two step design. A lot of design methods, most of which are basically an iterative and sequential design of the plant and the controller, have been proposed for the integrated design problem and each of them showed the advantage of its proposed design method over the conventional two step design scheme with their 
numerical examples (Onoda and Haftka 1987, Rao et al. 1988, Kajiwara et al. 1994, Grigoriadis et al. 1996, Hiramoto et al. 2000, Hiramoto and Doki 2004). However, unfortunately all integrated design problems result in a kind of BMI problem even if we assume that the coefficient matrices of the plant state space (or descriptor) form are linear functions on structural design parameters (Tanaka and Sugie 1998). It is well known that the BMI problem is an NP hard problem, i.e., roughly speaking, there are no efficient methods to obtain the global optimal solution, in contrast these for LMI problem (Blondel and Tsitsiklis 2000). In the integrated design problem some researches obtained a local optimal pair of the plant and the controller by sequential LMI optimization for the formulated BMIs (Tanaka and Sugie 1998, Chien 2000, Lu and Skelton 2000). However, these methods required all coefficient matrices of the state space (or the descriptor) form of the plant to be linear functions on the structural design parameter. In general, the structural design parameter enters the matrices in a non-linear manner and the methods are not capable in such a general case. Note that Iwasaki et al. (2000) proposed a plant design framework based on a finite frequency positive realness property and derived a design condition in terms of LMIs. However it could only change zeros of the system, not poles. Furthermore it also required the coefficient matrices of the state-space form to be linear functions on structural design parameters.

In this paper we propose an integrated design method with a homotopy approach to optimize the structural and control design parameters simultaneously. The homotopy technique has been applied to some control system design problems which are formulated as BMI problems (Hassibi et al. 1999, Zhai et al. 2001, Mehendale and Grigoriadis 2003) and has shown successful results numerically. In this paper the homotopy method is applied to the matrix inequalities, which originally were LMIs in the case of controller synthesis problem, representing the norm constraints of the closed-loop system. The overview of the proposed method is presented as follows. First, the optimal controller is obtained for a fixed plant with the LMIs representing the closed-loop specification(s). This is a convex optimization problem. Second, we assume a small amount of perturbations both for the coefficient matrices of the state space form of the plant, matrices related to the controller and the performance index in the LMIs. The obtained conditions are no longer LMIs on those perturbations. However, in the case where those perturbations are sufficiently small, we can approximate the matrix inequalities to LMIs on the perturbations by ignoring their second or the higher order products. Third, those approximated LMIs are solved with standard LMI solvers to obtain the optimal perturbations with a constraint such that the perturbation of the performance index becomes less than zero. The plant is updated to the new one by incorporating the obtained perturbation and go back to the first step. The above algorithm is iterated until a kind of convergence is obtained. In this design algorithm we can show a local convergence of the design parameters. The details of the design method will be mentioned later.

The advantages of the proposed design method are enumerated as follows.

- We can employ all LMIs, which have been obtained for the controller synthesis, for the integrated design. This means that we can utilize the variety of the LMI conditions for the controller design problem. In fact, we will consider a multiobjective problem like the $\mathcal{H}_{2} / \mathcal{H}_{\infty}$ problem given in Scherer et al. (1997) in the design example. There are no additional factors of the conservatism that comes from the integrated design formulation itself in the proposed method.

- The obtained closed-loop system is always optimal in the sense of the LMIs of interest in each iteration because the optimal controller is computed for all perturbed plant by solving the same LMIs obtained with the fixed plant. This means that we can obtain the optimal perturbation of the plant design parameter in the neighbourhood of the optimal closed-loop system and are able to obtain a better local optimal solution.

- The non-linear dependence of the plant state space matrices on the structural design parameters can be dealt with by approximating the non-linear dependence to a linear function. The approximation is simply carried out by taking the first order term of the series expansion of the non-linear function. The simple approximation scheme is always valid because we can restrict the amount of the perturbation arbitrarily small in the proposed design method such that the linear approximation of the non-linear function has a sufficient accuracy. (The price for the limitation is increasing the number of iterations for the convergence. The numerical example will be presented to describe the details.) With this advantage we can deal with a more general class of the integrated design problem whose structural design parameters enter non-linearly to the plant state space matrices.

Those advantages of the proposed design method will be demonstrated in design examples.

The rest of the paper is organized as follows: in $\S 2$, the integrated design problem of structural and control system is formulated; the homotopy-like design algorithm is presented in $\S 3$. Several features of the proposed design method are also stated in this section. Various design examples to show the effectiveness of the 
proposed design scheme are given in $\S 4$. In $\S 5$, the conclusion of this paper is presented.

\section{Integrated design problem}

The control object is represented as the following general state space form:

$$
\left\{\begin{array}{l}
\dot{x}(t)=A(p) x(t)+B_{1}(p) w(t)+B_{2}(p) u(t) \\
z(t)=C_{1}(p) x(t)+D_{11}(p) w(t)+D_{12}(p) u(t) \\
y(t)=C_{2}(p) x(t)+D_{21}(p) w(t),
\end{array}\right.
$$

where $x(t) \in \mathbf{R}^{n x}, w(t) \in \mathbf{R}^{n w}, u(t) \in \mathbf{R}^{n u}, z(t) \in \mathbf{R}^{n z}$ and $y(t) \in \mathbf{R}^{n y}$ are the state vector, the disturbance, and the control effort, the controlled output and the measurement respectively. Matrices $A(p), B_{1}(p), B_{2}(p), C_{1}(p)$, $C_{2}(p), D_{11}(p), D_{12}(p)$ and $D_{21}(p)$ have conformable dimensions and are (not necessarily linear) functions of $n p$ dimensional structural design parameter vector denoted as $p:=\left[p_{1}, \ldots, p_{n p}\right]^{T}$. In mechanical systems to be controlled, the damping, the stiffness and the sensor/ actuator placement, etc. are supposed to be elements of vector $p$. In this paper vector $p$ is assumed to be a real vector in a set $\mathcal{P}$ defined as the following:

$$
\mathcal{P}:=\left\{p \in \mathbf{R}^{n p}: p_{l} \leq p \leq p_{u}, p_{l}, p_{u} \in \mathbf{R}^{n x}\right\},
$$

where $p_{l}$ and $p_{u}$ are the lower and the upper bounds of the vector $p$ respectively.

For the control object possessing the adjustable design parameters given in (1) a following full order feedback controller is synthesised:

$$
\left\{\begin{array}{l}
\dot{x}_{K}(t)=A_{K} x_{K}(t)+B_{K} y(t) \\
u(t)=C_{K} x_{K}(t),
\end{array}\right.
$$

where $x_{K}(t) \in \mathbf{R}^{n x}$ is the state vector of the controller and all coefficient matrices in (3) have appropriate dimensions. Let the transfer function matrix of the controller as $K(s)$. Note that those coefficient matrices also can be considered as functions on the structural design parameter vector $p$.

The closed-loop system with the plant in (1) and the controller in (3) is given as

$$
\begin{gathered}
\left\{\begin{array}{l}
\dot{x}_{c l}(t)=A_{c l} x_{c l}(t)+B_{c l} w(t) \\
z(t)=C_{c l} x_{c l}(t)+D_{c l} w(t),
\end{array}\right. \\
x_{c l}(t):=\left[\begin{array}{c}
x(t) \\
x_{K}(t)
\end{array}\right], \quad A_{c l}:=\left[\begin{array}{cc}
A & B_{2} C_{K} \\
B_{K} C_{2} & A_{K}
\end{array}\right], \\
B_{c l}:=\left[\begin{array}{c}
B_{1} \\
B_{K} D_{21}
\end{array}\right], \quad C_{c l}:=\left[\begin{array}{ll}
C_{1} & D_{12} C_{K}
\end{array}\right], \quad D_{c l}:=D_{11} .
\end{gathered}
$$

Define the transfer function matrix of the closed-loop system (4) as $G_{c l}(s)$. For the closed-loop system we define a scalar performance index $J$ as

$$
J:=\left\|G_{c l}(s)\right\|_{\bullet}
$$

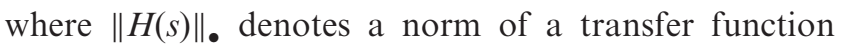
$H(s)$. We can assign the performance index $J$ for various types of norm of $G_{c l}(s)$, e.g., $\mathcal{H}_{2}, \mathcal{H}_{\infty}$ and $\mathcal{L}_{\infty}$ norm etc. or the weighted sum of such closed-loop norms (multiobjective case). Now the integrated design problem of the structural and control systems is formulated as the following:

Integrated design problem of structural and control systems: Find the structural design parameter $p_{\text {opt }} \in \mathcal{P}$ and the controller $K_{\text {opt }}(s)$ which minimize $J$ or satisfy $J \leq J_{u}\left(J_{u}>0\right)$ where $J_{u}$ is a scaler representing the performance specification determined by the designer.

Remark 1: In the case of the multiobjective problem, it is often the case of minimizing a norm of a closed-loop transfer function from the components of $w(s):=$ $\mathcal{L}(w(t))$ to those of $z(s):=\mathcal{L}(z(t))$ subject to inequality constraints on other norms of another closed-loop transfer matrix from (other) components of $w(s)$ to (other) ones of $z(s)$. The formulated problem is also capable in this situation.

Remark 2: In some cases we need to optimize or constrain the open-loop plant for a structural objective function by adjusting the $p \in \mathcal{P}$. We can also deal with such a structural optimization problem without any difficulties.

\section{Homotopy like design algorithm}

\subsection{LMI conditions for controller design}

The problem formulation in the previous section does not assume the specific norm of the closed-loop system. Actually we can deal with all kinds of norm if the optimal controller synthesis conditions are given as LMIs on controller design parameters (Scherer et al. 1997). In this subsection we introduce LMI conditions for the optimal $\mathcal{H}_{2}$ and $\mathcal{H}_{\infty}$ controllers, as examples, to mention the following homotopy like method concretely. Furthermore, we show the principle for a design of a multiobjective controller, say, $\mathcal{H}_{2} / \mathcal{H}_{\infty}$ controller, in the sense of a sufficient condition by taking common Lyapunov matrices that appear in LMI conditions on single objective problem, i.e., each of $\mathcal{H}_{2}$ or $\mathcal{H}_{\infty}$.

$\mathcal{H}_{2}$ norm (Scherer et al. 1997): A controller $K_{2}(s)$ yielding the $\left\|G_{c l}(s)\right\|_{2}^{2}<\gamma_{2}\left(\gamma_{2}>0\right)$ exists if and only if the following LMIs have solution matrices $X_{2}=X_{2}^{T}$, $Y_{2}=Y_{2}^{T} \in \mathbf{R}^{n x \times n x}, \quad Q=Q^{T} \in \mathbf{R}^{n z \times n z}, \quad \hat{A}_{2} \in \mathbf{R}^{n x \times n x}$, 
$\hat{B}_{2} \in \mathbf{R}^{n x \times n y}$ and $\hat{C}_{2} \in \mathbf{R}^{n u \times n x}$ :

$$
\begin{aligned}
& {\left[\begin{array}{lll}
\Theta_{11} & \Theta_{21}^{T} & \Theta_{31}^{T} \\
\Theta_{21} & \Theta_{22} & \Theta_{32}^{T} \\
\Theta_{31} & \Theta_{32} & \Theta_{33}
\end{array}\right]<0,} \\
& {\left[\begin{array}{lll}
\Lambda_{11} & \Lambda_{21}^{T} & \Lambda_{31}^{T} \\
\Lambda_{21} & \Lambda_{22} & \Lambda_{32}^{T} \\
\Lambda_{31} & \Lambda_{32} & \Lambda_{33}
\end{array}\right]>0,}
\end{aligned}
$$

$$
\operatorname{trace}(Q)<\gamma_{2}, \quad D_{c l}=0
$$

where

$$
\begin{aligned}
& \Theta_{11}=A X_{2}+X_{2} A^{T}+B_{2} \hat{C}_{2}+\left(B_{2} \hat{C}_{2}\right)^{T}, \\
& \Theta_{21}=\hat{A}_{2}+A^{T}, \\
& \Theta_{22}=A^{T} Y_{2}+Y_{2} A+\hat{B}_{2} C_{2}+\left(\hat{B}_{2} C_{2}\right)^{T}, \\
& \Theta_{31}=B_{1}^{T}, \quad \Theta_{32}=\left(Y_{2} B_{1}+\hat{B}_{2} D_{21}\right)^{T}, \quad \Theta_{33}=-I, \\
& \Lambda_{11}=X_{2}, \quad \Lambda_{21}=I, \quad \Lambda_{22}=Y_{2}, \quad \Lambda_{31}=C_{1} X_{2}+D_{12} \hat{C}_{2}, \\
& \Lambda_{32}=C_{1}^{T}, \quad \Lambda_{33}=Q .
\end{aligned}
$$

$\mathcal{H}_{\infty}$ norm (Scherer et al. 1997): A controller $K_{\infty}(s)$ yielding the $\left\|G_{c l}(s)\right\|_{\infty}<\gamma_{\infty}\left(\gamma_{\infty}>0\right)$ exists if and only if the following LMIs have solution matrices $X_{\infty}=X_{\infty}^{T}$, $Y_{\infty}=Y_{\infty}^{T} \in \mathbf{R}^{n x \times n x}, \quad \hat{A}_{\infty} \in \mathbf{R}^{n x \times n x}, \quad \hat{B}_{\infty} \in \mathbf{R}^{n x \times n y}$ and $\hat{C}_{\infty} \in \mathbf{R}^{\infty u \times n x}$ :

$$
\begin{gathered}
{\left[\begin{array}{llll}
\Xi_{11} & \Xi_{21}^{T} & \Xi_{31}^{T} & \Xi_{31}^{T} \\
\Xi_{21} & \Xi_{22} & \Xi_{32}^{T} & \Xi_{42}^{T} \\
\Xi_{31} & \Xi_{32} & \Xi_{33} & \Xi_{43}^{T} \\
\Xi_{41} & \Xi_{42} & \Xi_{43} & \Xi_{44}
\end{array}\right]<0,} \\
{\left[\begin{array}{cc}
X_{\infty} & I \\
I & Y_{\infty}
\end{array}\right]>0,}
\end{gathered}
$$

where

$$
\begin{aligned}
& \Xi_{11}=A X_{\infty}+X_{\infty} A^{T}+B_{2} \hat{C}_{\infty}+\left(B_{2} \hat{C}_{\infty}\right)^{T}, \\
& \Xi_{21}=\hat{A}_{\infty}+A^{T}, \\
& \Xi_{22}=A^{T} Y_{\infty}+Y_{\infty} A+\hat{B}_{\infty} C_{2}+\left(\hat{B}_{\infty} C_{2}\right)^{T}, \\
& \Xi_{31}=B_{1}^{T}, \quad \Xi_{32}=\left(Y_{\infty} B_{1}+\hat{B}_{\infty} D_{21}\right)^{T}, \quad \Xi_{33}=-\gamma_{\infty} I, \\
& \Xi_{41}=C_{1} X_{\infty}+D_{12} \hat{C}_{\infty}, \quad \Xi_{42}=C_{1}, \\
& \Xi_{43}=D_{11}, \quad \Xi_{44}=-\gamma_{\infty} I .
\end{aligned}
$$

In both of the above cases, the coefficient matrices of the controller

$$
K_{i}(s):=\left[\begin{array}{c|c}
\left(A_{K}\right)_{i} & \left(B_{K}\right)_{i} \\
\hline\left(C_{K}\right)_{i} & 0
\end{array}\right] \quad(i=2 \text { or } \infty)
$$

is given as

$$
\begin{aligned}
\left(C_{K}\right)_{i}= & \hat{C}_{i} M_{i}^{-T}, \quad\left(B_{K}\right)_{i}=N_{i}^{-1} \hat{B}_{i} \\
\left(A_{K}\right)_{i}= & N_{i}^{-1}\left(\hat{A}_{i}-N_{i}\left(B_{K}\right)_{i} C X_{i}-Y_{i} B\left(C_{K}\right)_{i} M_{i}^{T}\right. \\
& \left.-Y_{i} A X_{i}\right) M_{i}^{-T}
\end{aligned}
$$

where matrices $M_{i} \in \mathbf{R}^{n x \times n x}$ and $N_{i} \in \mathbf{R}^{n x \times n x}$ are nonsingular square solutions to the following decomposition problem:

$$
I-X_{i} Y_{i}=M_{i} N_{i}^{T}, \quad i=2 \text { or } \infty .
$$

Note that the above decomposition problem always has solution matrices $M_{i}$ and $N_{i}(i=2$ or $\infty)$ if the LMI for each controller design problem is feasible. Both of the above LMIs can be solved efficiently with a standard LMI solver, e.g. Gahinet et al. (1995).

In the case of the multiobjective problem of the above two criteria, i.e., the $\mathcal{H}_{2} / \mathcal{H}_{\infty}$ problem, the necessary and sufficient condition for the existence of controller $K_{2 / \infty}(s)$ yielding $\left\|G_{c l}(s)\right\|_{2}^{2}<\gamma_{2}$ and $\left\|G_{c l}(s)\right\|_{\infty}<\gamma_{\infty}$ is given by taking the common matrix variables as $\hat{A}_{2}=\hat{A}_{\infty}:=\hat{A}_{2 / \infty}, \hat{B}_{2}=\hat{B}_{\infty}:=\hat{B}_{2 / \infty}$ and $\hat{C}_{2}=\hat{C}_{\infty}:=$ $\hat{C}_{2 / \infty}$ in (6)-(8) and (9). (In this case the condition in (10) is automatically accomplished if the condition in (7) is feasible.) However those matrix inequality conditions are no longer LMIs in this case and the optimal $\mathcal{H}_{2} / \mathcal{H}_{\infty}$ controller design problem is still an open problem. For a sufficient condition to exist the controller satisfying $\left\|G_{c l}(s)\right\|_{2}^{2}<\gamma_{2}$ and $\left\|G_{c l}(s)\right\|_{\infty}<\gamma_{\infty}$ is obtained with the above variables changing and taking common Lyapunov matrices, i.e., $X_{2}=X_{\infty}=X_{2 / \infty}$ and $Y_{2}=$ $Y_{\infty}=Y_{2 / \infty}$ (Scherer et al. 1997, Lu and Skelton 2000). Then matrix inequality conditions in (6)-(8) and (9) are LMIs on each matrix variable and can be solved by LMI solvers. The resulting controller may be conservative depending on the given problem. However, this method currently can be said to be a reasonable way to obtain the multiobjective controller for the following reasons:

- We can obtain the controller taking multiple closedloop criteria (although it is not necessarily the optimal controller) efficiently because the conditions are given as LMIs on variable matrices.

- The order of the controller is always same as the plant in contrast to the method based on coprime factor technique (Boyd and Barratt 1991). The property of the controller order is favourable in real applications.

In this paper we take the above common Lyapunov matrix strategy in the case of a multiobjective problem even with other closed-loop norm constraints (e.g., $\mathcal{L}_{\infty}$ or other quadratic constraints) are considered. 
Remark 1: Recently a dilated-LMI approach for a multiobjective controller synthesis was proposed (Ebihara and Hagiwara 2004). They showed that the dilated-LMI technique is less conservative than the above common Lyapunov matrix approach and provided a controller synthesis condition in terms of LMIs for a specific multiobjective control problem $\left(\mathcal{H}_{2} / \mathcal{D}\right.$-stability problem). If the control specification is given as the closed-loop $\mathcal{H}_{2} / \mathcal{D}$-stability criterion we can employ this dilated-LMI based condition in the homotopy-like integrated design procedure which will be presented in the next subsection. However, because the $\mathcal{H}_{\infty}$ synthesis problem for continuous time systems is still open in the dilated-LMI framework (Ebihara and Hagiwara 2004) and we deal with the $\mathcal{H}_{2} / \mathcal{H}_{\infty}$ problem as a representative of various multiobjective control problems in this paper, we still adopt the common Lyapunov matrix strategy (Scherer et al. 1997) as the reasonable option for the multiobjective controller design. (In $\S 4$ an $\mathcal{H}_{2} / \mathcal{H}_{\infty}$ integrated design problem for a simple mechanical system will be considered.)

\subsection{Homotopy-like design algorithm}

In this subsection we propose a homotopy-like integrated design method based on LMI conditions in the previous subsection. We show the algorithm only in the case of the $\mathcal{H}_{\infty}$ problem. The method is exactly the same when we consider the $\mathcal{H}_{2}$ problem or the multiobjective problem.

Define $\Delta p:=\left[\Delta p_{1}, \ldots, \Delta p_{n p}\right]^{T} \in \mathbf{R}^{n p}, \Delta \hat{A}_{\infty} \in \mathbf{R}^{n x \times n x}$, $\Delta \hat{B}_{\infty} \in \mathbf{R}^{n x \times n y}, \quad \Delta \hat{C}_{\infty} \in \mathbf{R}^{n u \times n x}, \quad \Delta X_{\infty}=\Delta X_{\infty}^{T} \in$ $\mathbf{R}^{n x \times n x}, \Delta Y_{\infty}=\Delta Y_{\infty}^{T} \in \mathbf{R}^{n x \times n x}$ and a scalar $\Delta \gamma_{\infty}$ as perturbations of $p, \hat{A}_{\infty}, \hat{B}_{\infty}, \hat{C}_{\infty}, X_{\infty}, Y_{\infty}$ and $\gamma_{\infty}$ in (9) and (10) respectively. With those perturbations define the perturbed version of (9) and (10) as follows:

$$
\begin{aligned}
& {\left[\begin{array}{llll}
\Phi_{11} & \Phi_{21}^{T} & \Phi_{31}^{T} & \Phi_{31}^{T} \\
\Phi_{21} & \Phi_{22} & \Phi_{32}^{T} & \Phi_{42}^{T} \\
\Phi_{31} & \Phi_{32} & \Phi_{33} & \Phi_{43}^{T} \\
\Phi_{41} & \Phi_{42} & \Phi_{43} & \Phi_{44}
\end{array}\right]<0,} \\
& {\left[\begin{array}{cc}
X_{\infty}+\Delta X_{\infty} & I \\
I & Y_{\infty}+\Delta Y_{\infty}
\end{array}\right]>0}
\end{aligned}
$$

where

$$
\begin{gathered}
\Phi_{11}=A(p+\Delta p)\left(X_{\infty}+\Delta X_{\infty}\right)+\left(X_{\infty}+\Delta X_{\infty}\right) A(p+\Delta p)^{T} \\
+B_{2}(p+\Delta p)\left(\hat{C}_{\infty}+\Delta C_{\infty}\right)+\left(\hat{C}_{\infty}+\Delta C_{\infty}\right)^{T} \\
\times B_{2}(p+\Delta p)^{T} \\
\Phi_{21}=\hat{A}_{\infty}+\Delta \hat{A}_{\infty}+A(p+\Delta p)^{T},
\end{gathered}
$$

$$
\begin{gathered}
\Phi_{22}=A(p+\Delta p)^{T}\left(Y_{\infty}+\Delta Y_{\infty}\right)+\left(Y_{\infty}+\Delta Y_{\infty}\right) A(p+\Delta p) \\
+\left(\hat{B}_{\infty}+\Delta \hat{B}_{\infty}\right) C_{2}(p+\Delta p)+C_{2}(p+\Delta p)^{T} \\
\times\left(\hat{B}_{\infty}+\Delta \hat{B}_{\infty}\right)^{T} \\
\Phi_{31}=B_{1}(p+\Delta p)^{T}, \\
\Phi_{32}=\left\{\left(Y_{\infty}+\Delta Y_{\infty}\right) B_{1}(p+\Delta p)+\left(\hat{B}_{\infty}+\Delta \hat{B}_{\infty}\right)\right. \\
\left.\times D_{21}(p+\Delta p)\right\}^{T}, \\
\quad \Phi_{33}=-\left(\gamma_{\infty}+\Delta \gamma_{\infty}\right) I, \\
\Phi_{41}=C_{1}(p+\Delta p)\left(X_{\infty}+\Delta X_{\infty}\right)+D_{12}(p+\Delta p) \\
\times\left(\hat{C}_{\infty}+\Delta \hat{C}_{\infty}\right), \\
\Phi_{42}=C_{1}(p+\Delta p), \quad \Phi_{43}=D_{11}(p+\Delta p), \\
\Phi_{44}=-\left(\gamma_{\infty}+\Delta \gamma_{\infty}\right) I .
\end{gathered}
$$

If all coefficient matrices in (1) are linear functions on $p$, the perturbed coefficient matrices, say, $A(p+\Delta p)$, $B_{1}(p+\Delta p)$ and $B_{2}(p+\Delta p)$ etc. are given explicitly as

$$
\star(p+\Delta p)=\star+\sum_{j=1}^{n p} \Delta p_{j} \frac{\partial \star}{\partial p_{j}},
$$

where the symbol $\star$ denotes the component of each coefficient matrix in (1). If a component of those coefficient matrices is a non-linear function on $p$, equation (13) is satisfied approximately if $\Delta p$ is sufficiently small such that the linear approximation, obtained by taking the first order term of the infinite series expansion of the non-linear function, has a certain accuracy. We denote those (approximated) perturbations of coefficient matrices in (1) as $\Delta A, \Delta B_{1}, \Delta B_{2}$, $\Delta C_{1}, \Delta C_{2}, \Delta D_{11} \Delta D_{12}$ and $\Delta D_{21}$ respectively. Then, all components of (11) can be expanded in the form of the second order equations in terms of perturbations. Obviously (11) gives BMIs on those perturbations and is difficult to solve. However, if we assume all perturbations are small, it is valid to approximate those BMIs as LMIs by ignoring the 2nd order product of perturbation matrices. We take those approximated LMIs for the integrated design. The linear approximation of BMI in (11) is given as the following:

$$
\left[\begin{array}{llll}
\Psi_{11} & \Psi_{21}^{T} & \Psi_{31}^{T} & \Psi_{31}^{T} \\
\Psi_{21} & \Psi_{22} & \Psi_{32}^{T} & \Psi_{42}^{T} \\
\Psi_{31} & \Psi_{32} & \Psi_{33} & \Psi_{43}^{T} \\
\Psi_{41} & \Psi_{42} & \Psi_{43} & \Psi_{44}
\end{array}\right]<0
$$


where

$$
\begin{aligned}
\Psi_{11}= & A X_{\infty}+X_{\infty} A^{T}+B_{2} \hat{C}_{\infty}+\hat{C}_{\infty}^{T} B_{2}^{T}+A \Delta X_{\infty} \\
+ & \Delta X_{\infty} A^{T}+\Delta A X_{\infty}+X_{\infty} \Delta A^{T}+B_{2} \Delta \hat{C}_{\infty} \\
+ & \Delta \hat{C}_{\infty}^{T} B_{2}^{T}+\Delta B_{2} \hat{C}_{\infty}+\hat{C}_{\infty}^{T} \Delta B_{2}^{T}, \\
& \Psi_{21}=\hat{A}_{\infty}+\Delta \hat{A}_{\infty}+(A+\Delta A)^{T}, \\
\Psi_{22}= & A^{T} Y_{\infty}+Y_{\infty} A+\hat{B}_{\infty} C_{2}+C_{2}^{T} \hat{B}_{\infty}^{T}+A^{T} \Delta Y_{\infty} \\
+ & \Delta Y_{\infty} A+\Delta A^{T} Y_{\infty}+Y_{\infty} \Delta A+\hat{B}_{\infty} \Delta C_{2} \\
+ & \Delta C_{2}^{T} \hat{B}_{\infty}^{T}+\Delta \hat{B}_{\infty} C_{2}+C_{2}^{T} \Delta \hat{B}_{\infty}^{T}, \\
& \Psi_{31}=\left(B_{1}+\Delta B_{1}\right)^{T}, \\
\Psi_{32}= & B_{1}^{T} Y_{\infty}+D_{21}^{T} \hat{B}_{\infty}^{T}+B_{1}^{T} \Delta Y_{\infty}+\Delta B_{1}^{T} Y_{\infty} \\
& +D_{21}^{T} \Delta \hat{B}_{\infty}^{T}+\Delta D_{21}^{T} \hat{B}_{\infty}^{T}, \\
\Psi_{33}= & \Phi_{33}, \\
\Psi_{41}= & C_{1} X_{\infty}+D_{12} \hat{C}_{\infty}+C_{1} \Delta X_{\infty} \\
& +\Delta C_{1} X_{\infty}+D_{12} \Delta \hat{C}_{\infty}+\Delta D_{12} \hat{C}_{\infty} \\
\Psi_{42}= & C_{1}+\Delta C_{1}, \quad \Psi_{43}=D_{11}+\Delta D_{11}, \quad \Psi_{44}=\Phi_{44} .
\end{aligned}
$$

Equations (14) and (12) are LMIs on all perturbation matrices and can be solved efficiently.

As mentioned before the linear approximation of BMIs is reasonable only if the perturbations are small. Hence, we need to confine the amount of those perturbations in some sense. In this paper we take a constraint on the perturbation matrices given by

$$
\bar{\sigma}(\Delta *)<\epsilon \bar{\sigma}(*) \quad(\epsilon>0),
$$

where $\bar{\sigma}(*)$ denotes the maximum singular value of a variable matrix, e.g., $p, X_{\infty}$ and $Y_{\infty}$ etc. The constraint in (15) is also given as the following LMI:

$$
\left[\begin{array}{cc}
\epsilon \bar{\sigma}(*) I & \Delta * \\
(\Delta *)^{T} & \epsilon \bar{\sigma}(*) I
\end{array}\right]>0 .
$$

Therefore, we can obtain the small amount of the optimal perturbations by combining LMI conditions (14), (12) and (16). With the above linearized LMIs we propose the following integrated design algorithm.

Algorithm 1 (Integrated design algorithm):

Step 0: Define the iteration number $i=0$. Set the initial value of the structural design parameter vector $p^{0} \in \mathcal{P}$. In the following we define superscript ' $i$ ' which denotes the ith iteration of this algorithm.

Step 1: Set $\epsilon>0$ in (15). For the plant derived for the fixed $p^{i} \in \mathcal{P}$ obtain the optimal $\mathcal{H}_{\infty}$ controller $K^{i}(s)$ and the performance index $J^{i}:=\gamma_{\infty}^{i}$ for the fixed plant with LMI conditions (9) and (10).
Step 2: Obtain the optimal perturbation matrices, e.g., $\Delta p^{i}, \Delta \hat{A}_{\infty}^{i}$ and $\Delta \hat{B}_{\infty}^{i}$ etc. minimizing the perturbation of the performance index $\Delta \gamma_{\infty}^{i}$ subject to constraints $\Delta \gamma_{\infty}^{i}<0$ and $p^{i}+\Delta p^{i} \in \mathcal{P}$, and satisfying (approximated) LMIs in (14), (12) and (16). If such perturbations can not be obtained, then set the optimal structural vector $p_{\text {opt }}:=p^{i}$ and $K_{\text {opt }}(s):=K^{i}(s)$ and stop. Otherwise, update $p^{i+1} \leftarrow p^{i}+\Delta p^{i}$ and go to the next step.

Step 3: Obtain the optimal $\mathcal{H}_{\infty}$ controller $K^{i+1}(s)$ and the performance index $J^{i+1}:=\gamma_{\infty}^{i+1}$ again for the plant yielding the new structural design parameter vector $p^{i+1}$. If $J^{i+1}-J^{i} \geq 0$ then $\epsilon \leftarrow \eta \epsilon$ where $0<\eta<1$ and go to Step 2. Otherwise update $p^{i} \leftarrow p^{i+1}$ and go to Step 1 .

In the proposed algorithm at least a convergence to a local optimal solution is assured by the iteration between Steps 2 and 3 because the structural design parameter $p$ is not updated unless the condition $J^{i+1}-J^{i}<0$ is satisfied. In this algorithm the value of $\epsilon$ in (15) is working like a step width of general descentbased optimization methods, e.g., steepest descent method etc. In the integrated design problem some design methods guaranteed the local convergence of the objective function have also been proposed (Tanaka and Sugie 1998, Chien 2000, Lu and Skelton 2000). However, in all of their design algorithms all coefficient matrices of state space (or descriptor) form must be linear functions on the structural design parameters. Meanwhile in our method the structural design parameters and the controller can be adjusted simultaneously with a guarantee of the local convergence even if the structural design parameters enter non-linearly in coefficient matrices of the plant state space form (1). Therefore by taking the proposed integrated design method we can largely extend the class of the plant to which the integrated design technique can be applied.

Let us make some remarks on the proposed algorithm.

Remark 2: According to the problem we can relax the condition in (15) on the component of the vector $p$ as long as we can obtain a decreasing sequence of the performance index $J^{i}$. From the authors' experience (as shown in §4) this relaxation is possible if all coefficient matrices of the plant state space form are linear functions on the vector $p$ and make the convergence much faster. If those coefficient matrices are not linear functions on the vector $p$, we must always set $\epsilon>0$ as a quite small value, say, $10^{-3}$ such that the linear approximation of the non-linear dependence mentioned before is valid. Currently we do not have 
a systematic way to select an appropriate value $\epsilon$ to obtain a good result, therefore, a kind of trial and error scheme may be required. These characteristics from the numerical aspect will be mentioned again in $\S 4$.

Remark 3: The above algorithm is specifically described in the case for minimizing the $\mathcal{H}_{\infty}$ norm of the closed-loop system. However, as mentioned before, we can deal with another closed-loop norm or a multiobjective problem by changing the above LMI conditions appropriately. The algorithm works as long as the optimal controller synthesis condition is given in terms of LMIs on controller related matrices.

Remark 4: In this paper we assume the full-order output feedback controller which does not have any specific structures. We note that we can also deal with the design problem in which the controller order is less than that of the plant (reduced-order controller design) (e.g., Iwasaki (1999)) and/or the controller must satisfy some structural properties like a decentralized control problem (e.g., Zhai et al. (2001), Mehendale and Grigoriadis (2003)) if there exists an efficient (analytical or numerically tractable) method to obtain such controllers. The integrated design algorithm proposed in this paper can embed the method to obtain such controllers. However, the above problems are generally still open and only some iterative algorithms for a locally optimal controller have been proposed so far. Therefore the amount of computation for the integrated design subject to the order and/or the structural constraints of the controller may become much larger than that of the current full-order case.

Remark 5: Rakowska et al. (1993) applied a homotopy method to an integrated design problem. They considered a specific performance index related to the integrated design of a space structure and discussed a homotopy-based solution procedure. In this study the performance index about the closed-loop performance is quite elementary and the control law is limited to a direct velocity feedback law which is only effective under the collocation of sensors and actuators. On the other hand we aim to propose a general solution procedure to obtain a solution to various integrated design problems which are formulated as BMIs. Those BMI conditions originally derive from LMI conditions for the controller design. Currently a large number of LMI conditions to obtain the controller with various properties are available. This means that we can deal with many closed-loop specifications by using the proposed homotopy-like integrated design method.

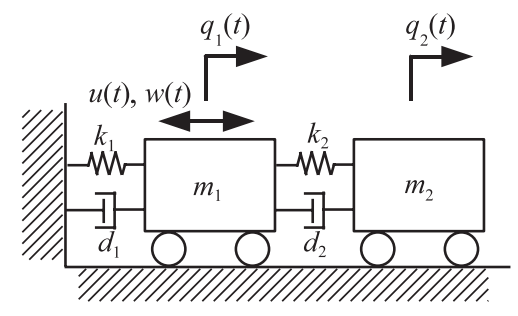

Figure 1. Active control of 2DOF system.

\section{Design examples}

\subsection{DOF system}

Let us consider an active control of a 2DOF system, depicted in figure 1, where $q_{1}(t), q_{2}(t), w(t)$ and $u(t)$ are the displacement of $m_{1}$ and $m_{2}$, the disturbance force and the control force respectively. The spring and damper denoted by $k_{i}$ and $d_{i}(i=1,2)$ connect between the fixed wall and $m_{1}$ and $m_{1}$ and $m_{2}$ respectively. The disturbance and the control force are applied to mass $m_{1}$ and the displacement of the mass $m_{2}$ is measured to suppress the vibration of the same mass. By taking the state vector $x(t)$ as $x(t):=\left[\begin{array}{llll}q_{1}(t) & q_{2}(t) & \dot{q}_{1}(t) & \dot{q}_{2}(t)\end{array}\right]^{T}$, the state space form in (1) is

$$
\begin{aligned}
& \left\{\begin{array}{l}
\dot{x}(t)=A x(t)+\left[\begin{array}{ll}
B_{1}^{1} & B_{1}^{2}
\end{array}\right]\left[\begin{array}{c}
w(t) \\
v(t)
\end{array}\right]+B_{2} u(t) \\
z(t)=\left[\frac{z_{1}(t)}{z_{2}(t)}\right]=\left[\frac{C_{1}^{1}}{0_{1 \times 4}}\right] x(t)+\left[\begin{array}{l}
0 \\
1
\end{array}\right] u(t) \\
y(t)=C_{2} x(t)+\left[\begin{array}{ll}
0 & 10^{-3}
\end{array}\right]\left[\begin{array}{c}
w(t) \\
v(t)
\end{array}\right],
\end{array}\right. \\
& A:=\left[\begin{array}{cccc}
0 & 0 & 1 & 0 \\
0 & 0 & 0 & 1 \\
-\frac{k_{1}+k_{2}}{m_{1}} & \frac{k_{2}}{m_{1}} & -\frac{d_{1}+d_{2}}{m_{1}} & \frac{d_{2}}{m_{1}} \\
\frac{k_{2}}{m_{2}} & -\frac{k_{2}}{m_{2}} & \frac{d_{2}}{m_{2}} & -\frac{d_{2}}{m_{2}}
\end{array}\right], \\
& B_{1}^{1}:=\left[\begin{array}{c}
0 \\
0 \\
\frac{1}{m} \\
0
\end{array}\right] \text {, } \\
& B_{1}^{2}:=0_{4 \times 1}, \quad B_{2}:=B_{1}^{1}, \quad C_{1}^{1}=\left[\begin{array}{llll}
0 & 10^{3} & 0 & 0
\end{array}\right], \\
& C_{2}=\left[\begin{array}{llll}
0 & 1 & 0 & 0
\end{array}\right],
\end{aligned}
$$

where $v(t)$ is the measurement noise.

We consider a multiobjective problem where the performance index $J_{m}$ is defined by

$$
J_{m}:=\overline{J_{\infty}}+\alpha \overline{J_{2}}, \quad \alpha>0,
$$




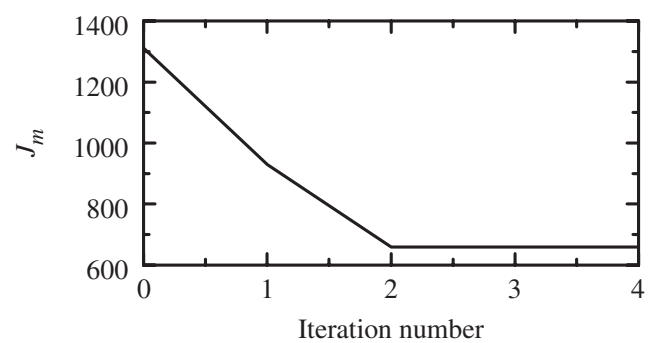

Figure 2. The optimization history for the performance index $J_{m}$.

where $\overline{J_{\infty}}$ and $\overline{J_{2}}$ are upper bounds of the closed-loop $\mathcal{H}_{\infty}$ and the square of $\mathcal{H}_{2}$ norm from $[w(t) v(t)]^{T}$ to $z_{1}(t)$ and $z_{2}(t)$ respectively. The scalar $\alpha>0$ is a weighting parameter between the above two performance indices. For the fixed plant, the controller optimizing $J_{m}$ can be obtained by taking $X_{2}=X_{\infty}=X, \quad Y_{2}=Y_{\infty}=Y$, $\hat{A}_{2}=\hat{A}_{\infty}=\hat{A}, \quad \hat{B}_{2}=\hat{B}_{\infty}=\hat{B} \quad$ and $\quad \hat{C}_{2}=\hat{C}_{\infty}=\hat{C} \quad$ in LMIs given as (6)-(8) and (9) and minimizing the $\gamma_{\infty}+\alpha \gamma_{2}$. In this example two cases of structural design parameter vectors are considered. Each result is shown in the following:

Case A: In this case we take the structural design parameter vector $p$ as:

$$
p:=\left[\begin{array}{ll}
d_{1} & k_{1}
\end{array}\right]^{T} .
$$

We set the lower and the upper bounds of the vector $p$ as $p_{l}:=10^{-2} \times\left[\begin{array}{ll}d_{1}^{n} & k_{1}^{n}\end{array}\right]^{T}$ and $p_{u}:=10 \times\left[\begin{array}{ll}d_{1}^{n} & k_{1}^{n}\end{array}\right]^{T}$, where $d_{1}^{n}=0.01(\mathrm{Ns} / \mathrm{m})$ and $k_{1}^{n}=1(\mathrm{~N} / \mathrm{m})$, respectively. From (18) only the matrix $A$ is a linear function of each component of the vector $p$ in this case. The other physical design parameters are defined as $m_{1}=m_{2}=1$ $(\mathrm{kg}), d_{2}=d_{1}^{n}$ and $k_{2}=k_{1}^{n}$ respectively.

By taking $\epsilon=0.2$ (in (15)) and $\alpha=1$, the proposed integrated design algorithm is applied to the problem. However, we do not impose the norm constraint on each perturbation of the vector $p$ in the this case (see Remark 2). The initial value of the vector $p$ is $p^{0}:=\left[\begin{array}{ll}d_{1}^{n} & k_{1}^{n}\end{array}\right]^{T}$. Optimization histories for the performance index $J_{m}$, the structural design parameters $d_{1}$ and $k_{1}$ are shown in figures 2, 3 and 4 respectively.

The damping coefficient $d_{1}$ and the spring constant $k_{1}$ converge to their upper bounds respectively after only four times iteration of the proposed integrated design algorithm. To check the quality of the obtained solution (as mentioned in the previous section we can guarantee only the local convergence of the design parameters), we carry out the search for the whole design space $\mathcal{P}:=\left\{p: p_{l} \leq p \leq p_{u}\right\}$ by computing the optimal controller at 2500 plants obtained by gridding each range $\left(d_{1}:\left[10^{-4}, 0.1\right], k_{1}:\left[10^{-2}, 10\right]\right)$ of structural design parameters to 50 points. The $3 \mathrm{D}$ plot of the result of the exhaustive search is shown in figure 5. Clearly, in this

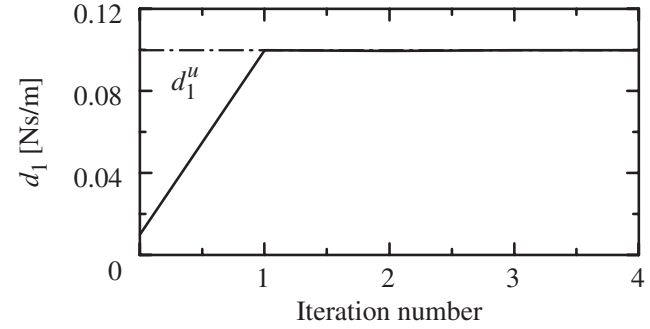

Figure 3. The optimization history for the damping coefficient $d_{1}$.

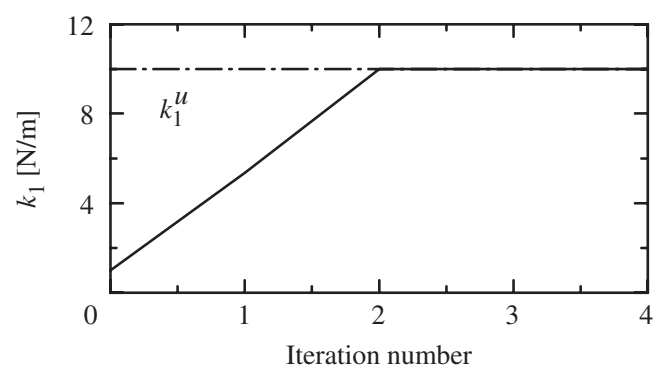

Figure 4. The optimization history for the spring constant $k_{1}$.

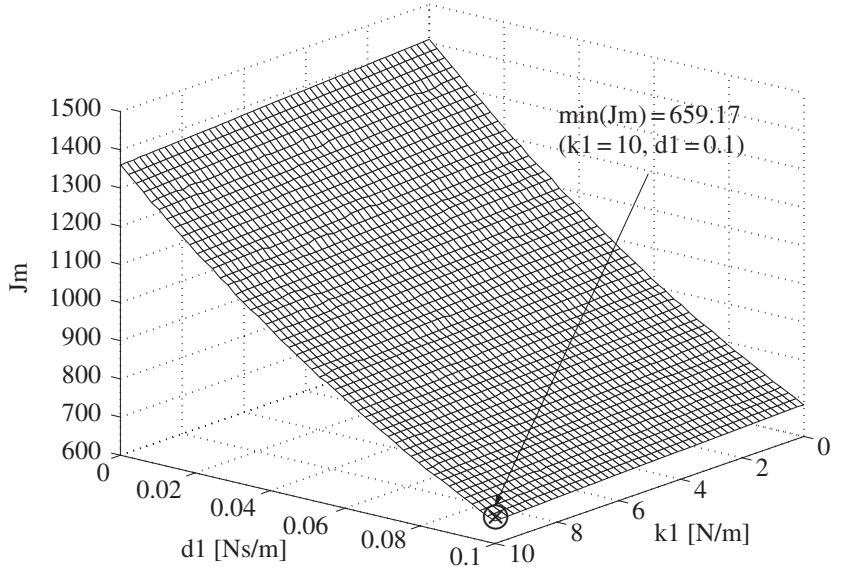

Figure 5. The actual performance index $J_{m}$ (Case A).

case we could successfully find out the global optimal solution with the proposed homotopy based method. This result also means that we could find out the global optimal solution of the current BMI problem. We conduct the optimization several times from other initial values which are randomly selected to check the convergence property of the design algorithm. Fortunately, we can obtain same global optimal solutions for almost all cases. Furthermore we carried out the optimization by imposing the norm constraint in (15) even on $\Delta p:=\left[\begin{array}{lll}\Delta d_{1} & \Delta k_{1}\end{array}\right]^{T}$. The optimal solution obtained was almost the same as in the case we showed. However, it took many more iterations to obtain. 


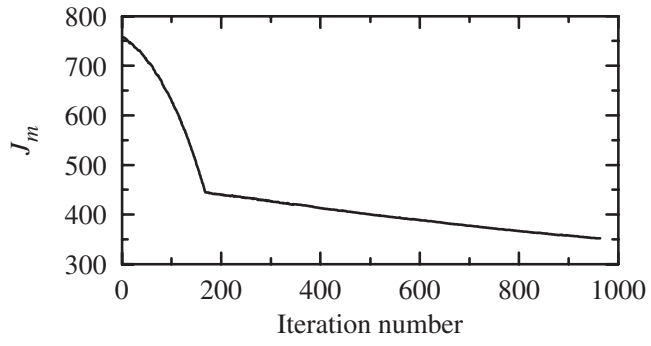

Figure 6. The optimization history for the performance index $J_{m}$ (mass optimization).

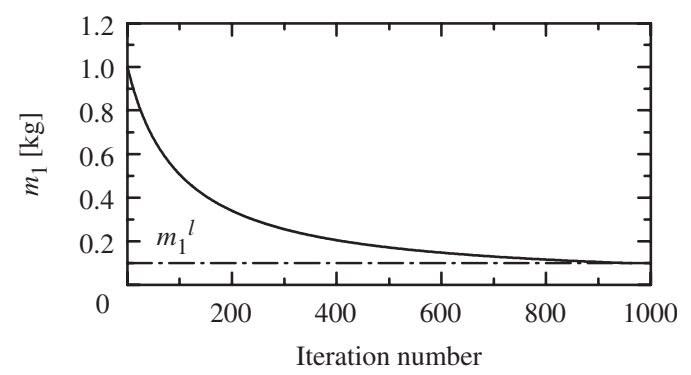

Figure 7. The optimization history for the mass $m_{1}$.

Case B: Vector $p$ is taken in this case to examine the non-linear dependence of the plant coefficient matrices on components of $p$ with

$$
p:=\left[\begin{array}{ll}
m_{1} & m_{2}
\end{array}\right]^{T} .
$$

The lower and the upper bounds of vector $p$ are specified $p_{l}:=0.1 \times\left[\begin{array}{ll}m_{1}^{n} & m_{2}^{n}\end{array}\right]$ and $p_{u}:=10 \times\left[\begin{array}{ll}m_{1}^{n} & m_{2}^{n}\end{array}\right]^{T}$, where $m_{1}^{n}=m_{2}^{n}=1(\mathrm{~kg})$ respectively. Obviously from (18), matrices $A, B_{1}$ and $B_{2}$ are non-linear functions on $m_{1}$ and $m_{2}$ in this case. Note that the methods proposed in Tanaka and Sugie (1998), Chien (2000) and $\mathrm{Lu}$ and Skelton (2000) can no longer handle this case because of the non-linear dependence of those coefficient matrices on vector $p$. For the same $\alpha$ as that of Case A, the optimal design is carried out. In this case we set $\epsilon=10^{-2}$ and the norm constraint in (15) is imposed on all perturbations. The result of the optimization is shown in figures 6-8. In contrast to Case $\mathrm{A}$ it takes around 900 time iterations to obtain the convergence.

In this case masses $m_{1}$ and $m_{2}$ are converged to their lower and upper bounds respectively. An exhaustive search is also conducted in the whole design parameter space $\mathcal{P}$ by taking the $50 \times 50$ gridding on the range $m_{1}$ : $[0.1,10]$ and $m_{2}:[0.1,10]$. The result is shown in figure 9 . We could successfully find out the global optimal solution again even in the case that the coefficient matrices of plant state space form are non-linear functions on vector $p$. Although the proposed algorithm is not guaranteed to obtain the global optimal solution,

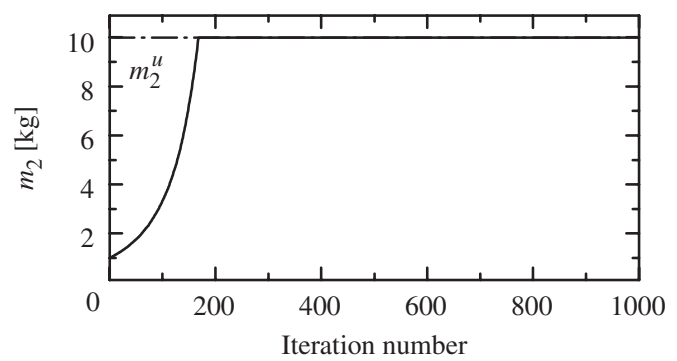

Figure 8. The optimization history for the mass $m_{2}$.

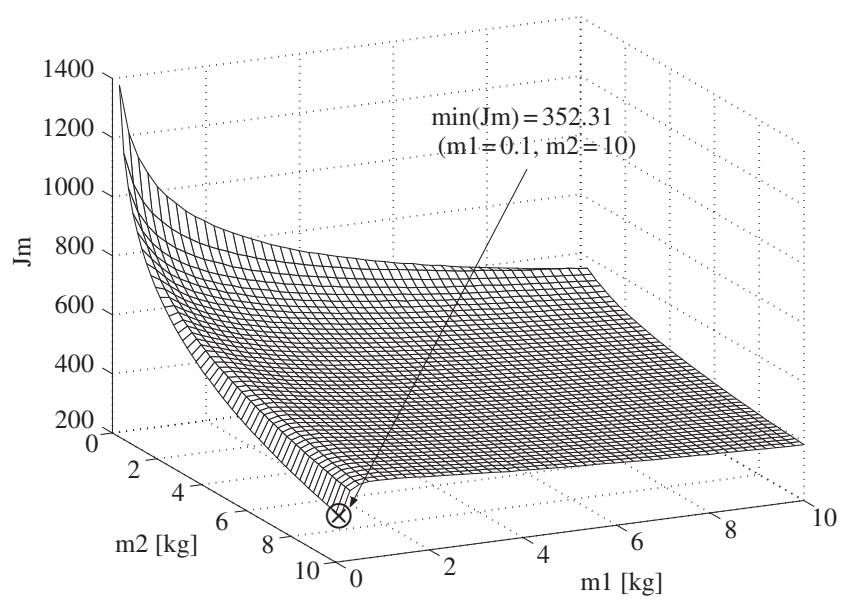

Figure 9. The actual performance index $J_{m}$ (Case B).

this result suggests the capability of the proposed design method for more general integrated design problems.

\subsection{Sensor/actuator placement}

A sensor/actuator placement problem is solved in this subsection. The optimal selection/placement of sensors and actuators is quite important in control system design because they strongly affect the achievable closed-loop performance. In some cases a convex optimization method exists for the optimal selection and/or placement of sensors and actuators and the corresponding optimal controller simultaneously (Geromel 1989, Oliveira and Geromel 2000). However, in general case optimal sensor/actuator selection and placement, the problem is still open and any analytical or numerically tractable methods to obtain the global optimal sensor/actuator selection and placement and the feedback controller have not been proposed so far. In this example we deal with an optimal sensor/actuator placement problem minimizing the $\mathcal{H}_{\infty}$ norm of the closed-loop system with the proposed homotopy-like design algorithm. 


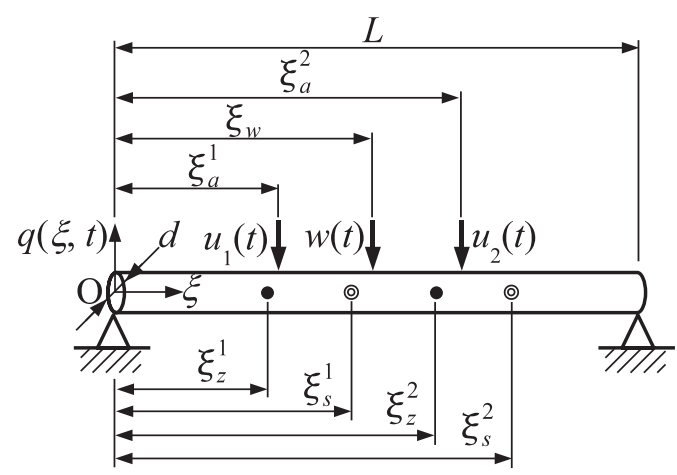

Figure 10. Simply supported beam system.

Let us consider an active vibration control system of a simply supported beam with a circular cross section in figure 10. The length, the diameter, the density and Young's modulus of the beam are denoted by $L(\mathrm{~m})$, $d(\mathrm{~m}), \rho\left(\mathrm{kg} / \mathrm{m}^{3}\right)$ and $E\left(\mathrm{~N} / \mathrm{m}^{2}\right)$ respectively. The moment of inertia of the area is obtained by $I:=\pi d^{4} / 64$. Two actuators producing control forces $u_{1}(t)$ and $u_{2}(t)$ are installed at $\xi=\xi_{a}^{1}, \xi=\xi_{a}^{2}$, respectively. Two sensors are equipped for measuring displacements $q\left(\xi_{s}^{1}, t\right)$ and $q\left(\xi_{s}^{2}, t\right)$. A disturbance force $w(t)$ is injected at $\xi=\xi_{w}$. We obtain the optimal sensor and actuator placement in this problem with the proposed method. The structural design parameter vector and its lower and upper bounds are defined by

$$
\begin{aligned}
p & :=\left[\begin{array}{llll}
\xi_{a}^{1} & \xi_{a}^{2} & \xi_{s}^{1} & \xi_{s}^{2}
\end{array}\right]^{T}, \quad p_{l}=0_{4 \times 1}, \\
p_{u} & =L \times\left[\begin{array}{llll}
1 & 1 & 1 & 1
\end{array}\right]^{T} .
\end{aligned}
$$

We assume that the displacement $q(\xi, t)$ can be approximated by

$$
q(\xi, t) \simeq \sum_{j=1}^{3} q_{j}(t) \phi_{j}(\xi),
$$

where $q_{j}(t)$ is the $j$ th modal displacement of the beam. The function $\phi_{i}(\xi)$ is a normalized $j$ th modal shape of a simply supported beam given as

$$
\phi_{j}(\xi)=\sqrt{\frac{2}{L}} \sin \left(\frac{j \pi \xi}{L}\right) .
$$

Then the (approximated) modal equation of motion of the beam system is obtained as

$$
\ddot{q}_{f}(t)+2 Z \Omega \dot{q}_{f}(t)+\Omega^{2} q_{f}(t)=L_{w} w(t)+L_{a} u(t),
$$

where $q_{f}(t):=\left[\begin{array}{lll}q_{1}(t) & q_{2}(t) & q_{3}(t)\end{array}\right]^{T}$ is the (approximated) modal displacement vector and $u(t):=$ $\left[\begin{array}{lll}u_{1}(t) & u_{2}(t)\end{array}\right]^{T}$ respectively. Matrices $Z:=\operatorname{diag}\left(\zeta_{1}, \zeta_{2}, \zeta_{3}\right)$ and $\quad \Omega:=\operatorname{diag}\left(\omega_{1}, \omega_{2}, \omega_{3}\right), \quad\left(\omega_{j}:=(j \pi)^{2} \sqrt{E I / \rho S L^{4}}\right.$, $\left.j=1,2,3, S=\pi d^{2} / 4\right)$ are the modal damping matrix
Table 1. Parameter values.

\begin{tabular}{lc}
\hline & Value \\
\hline Length $L(\mathrm{~m})$ & 1.00 \\
Diameter $d(\mathrm{~m})$ & $5.00 \times 10^{-3}$ \\
Density $\rho\left(\mathrm{kg} / \mathrm{m}^{3}\right)$ & $6.21 \times 10^{3}$ \\
Young's modulus $\left(\mathrm{N} / \mathrm{m}^{2}\right)$ & $6.06 \times 10^{6}$ \\
Proportional constant $\beta$ & $10^{-3}$ \\
Weighting $r_{1}$ and $r_{2}$ & 1 \\
\hline
\end{tabular}

and normal frequency matrix respectively. Matrices $L_{w}$ and $L_{a}$ are given as

$$
L_{w}:=\left[\begin{array}{l}
\phi_{1}\left(\xi_{w}\right) \\
\phi_{2}\left(\xi_{w}\right) \\
\phi_{3}\left(\xi_{w}\right)
\end{array}\right], \quad L_{a}:=\left[\begin{array}{ll}
\phi_{1}\left(\xi_{a}^{1}\right) & \phi_{1}\left(\xi_{a}^{2}\right) \\
\phi_{2}\left(\xi_{a}^{1}\right) & \phi_{2}\left(\xi_{a}^{2}\right) \\
\phi_{3}\left(\xi_{a}^{1}\right) & \phi_{3}\left(\xi_{a}^{2}\right)
\end{array}\right] .
$$

We take a controlled output $z(t)$ as

$$
z(t):=\left[\begin{array}{llll}
q(0.3 L, t) & q(0.6 L, t) & r_{1} u_{1}(t) & r_{2} u_{2}(t)
\end{array}\right]^{T},
$$

where $r_{1}$ and $r_{2}$ are the positive scalar weightings. By assuming the proportional damping, i.e., $Z=\beta \Omega$ $(0<\beta \ll 1)$ (Meirovitch 1990) and taking the state vector $x(t):=\left[\begin{array}{ll}q_{f}(t) & \dot{q}_{f}(t)\end{array}\right]^{T}$, we derive the following state space form of the beam system:

$$
\left\{\begin{array}{l}
\dot{x}(t)=A x(t)+B_{1}(t)+B_{2} u(t) \\
z(t)=C_{1} x(t)+D_{11} w(t)+D_{12} u(t), \\
y(t)=C_{2} x(t)+D_{21} w(t)
\end{array}\right.
$$

$$
\begin{aligned}
A & :=\left[\begin{array}{cc}
0_{3 \times 3} & I_{3} \\
-\Omega^{2} & -2 \beta \Omega^{2}
\end{array}\right], \quad B_{1}:=\left[\begin{array}{c}
0_{3 \times 1} \\
L_{w}
\end{array}\right], \quad B_{2}:=\left[\begin{array}{c}
0_{3 \times 2} \\
L_{a}
\end{array}\right], \\
C_{1} & :=\left[\begin{array}{cc}
L_{z} & 0_{2 \times 3} \\
0_{2 \times 6}
\end{array}\right], \quad D_{11}:=0_{4 \times 1}, \quad D_{12}:=\left[\begin{array}{c}
0_{2 \times 2} \\
\operatorname{diag}\left(r_{1}, r_{2}\right)
\end{array}\right], \\
C_{2} & :=\left[\begin{array}{ll}
L_{s} & 0_{2 \times 3}
\end{array}\right], \quad D_{21}:=0_{2 \times 1},
\end{aligned}
$$

where the matrices $L_{z}$ and $L_{s}$ are given as

$$
\begin{aligned}
L_{z} & :=\left[\begin{array}{lll}
\phi_{1}(0.3 L) & \phi_{2}(0.3 L) & \phi_{3}(0.3 L) \\
\phi_{1}(0.6 L) & \phi_{2}(0.6 L) & \phi_{3}(0.6 L)
\end{array}\right], \\
L_{s} & :=\left[\begin{array}{lll}
\phi_{1}\left(\xi_{s}^{1}\right) & \phi_{2}\left(\xi_{s}^{1}\right) & \phi_{2}\left(\xi_{s}^{1}\right) \\
\phi_{1}\left(\xi_{s}^{2}\right) & \phi_{2}\left(\xi_{s}^{2}\right) & \phi_{3}\left(\xi_{s}^{2}\right)
\end{array}\right] .
\end{aligned}
$$

In this case, matrices $B_{2}$ and $C_{2}$ are clearly non-linear functions on the vector $p$ in (22). We take the performance index (denoted by $J_{b}$ ) as the closed-loop $\mathcal{H}_{\infty}$ norm from $w(t)$ to $z(t)$. The optimal controller can be obtained for a fixed $p$ by minimizing $\gamma_{\infty}$ in LMIs in (9) and (10). The values of physical parameters are depicted in table 1 . Note that the material of the beam is assumed to be a kind of plastic which is used in Doki et al. (2002). 
Table 2. Optimal sensor/actuator placements.

\begin{tabular}{llccccc}
\hline Case & & $J_{b}$ & $\xi_{a}^{1}$ & $\xi_{a}^{2}$ & $\xi_{s}^{1}$ & $\xi_{s}^{2}$ \\
\hline I & Initial & 1.673 & 0.950 & 0.231 & 0.607 & 0.486 \\
& Optimal & 0.711 & 0.399 & 0.400 & 0.574 & 0.453 \\
II & Initial & 0.761 & 0.511 & 0.324 & 0.232 & 0.493 \\
& Optimal & 0.709 & 0.400 & 0.400 & 0.232 & 0.490 \\
III & Initial & 0.975 & 0.144 & 0.518 & 0.243 & 0.482 \\
& Optimal & 0.708 & 0.400 & 0.400 & 0.247 & 0.451 \\
IV & Initial & 2.574 & 0.550 & 0.493 & 0.030 & 0.219 \\
& Optimal & 0.709 & 0.399 & 0.401 & 0.030 & 0.229 \\
\hline
\end{tabular}

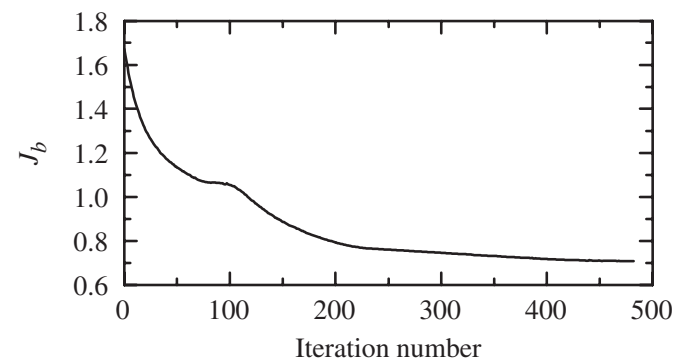

Figure 11. The optimization history for the performance index $J_{b}$ (case I).

By taking $\epsilon=5 \times 10^{-3}$, the proposed optimal design method is applied to the sensor/actuator placement problem. To obtain the better locally optimal solution, we conduct the optimization several times from different initial placements. In this example three local optimal solutions are obtained from six initial placements which are randomly selected. However, in most cases (in four cases from six initial placements) both actuator placements converge to the place where the disturbance is applied $(\xi=0.4 L)$. Those results are shown in table 2 with their initial placements. A typical result (case I in table 2) is presented in figures 11 and 12. In this problem, it is found that the performance index $J_{b}$ is quite insensitive to the sensor placements $\xi_{s}^{1}$ and $\xi_{s}^{2}$. As shown in table 2, we can see that the values of the optimal performance indices $\left(J_{b}\right)_{\text {opt }}$ are almost the same, irrespective of their sensor placements. This result also suggests we do not need to equip two actuators but need only one actuator at the place where the disturbance is injected.

We do not check whether the obtained solutions are globally optimal or not, in this example, because the computational load is too large to obtain the global optimal solution with the extensive search of the whole design parameter space. However we can claim that the obtained result is quite reasonable from the physical consideration because it is the efficient way to suppress

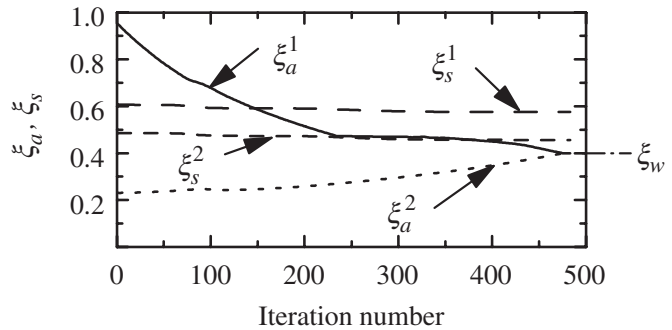

Figure 12. The optimization history for the sensor/actuator $\xi_{a}^{i}$ and $\xi_{s}^{i}(i=1,2)$ (Case I).

the effect of the disturbance force by collocating actuators.

The results of this section show that the proposed integrated design method works quite effectively even for the problems which earlier proposed BMI based integrated design approaches (Tanaka et al. 1998, Chien 2000, Lu and Skelton 2000) cannot deal with. The result of design examples indicates the capability of the proposed design scheme for more general and complex design problems in real applications.

The computational time to obtain the optimal solution in each example is discussed. In Case A of the 2DOF system example the computational time is less than one minute with a standard Windows OS PC (CPU: Pentium III $600 \mathrm{MHz}, 256 \mathrm{MB}$ RAM) with MATLAB software. In Case B of the 2DOF system and sensor/actuator placement examples, the time to obtain the optimal solution is up to several hours with the same PC. If a more sophisticated computer, which is currently available, is employed the computational time must be dramatically reduced and this fact indicates that the application of the proposed homotopy-like integrated design method to real industrial applications is now becoming more realistic.

\section{Conclusion}

We have investigated the homotopy based integrated design method in this paper. An iterative design algorithm based on the homotopy method has been proposed. In this method we can utilize all LMI conditions for controller synthesis to the integrated design problem. This fact means that we can deal with the multiobjective problem in the integrated design problem. Currently the LMI based approach is recognized as one of the most effective solution methods. The proposed method can also be applied to the case where the coefficient matrices of the plant state space form are non-linear functions on their structural design parameters by taking the linear approximation of the non-linear dependence and limiting the amount of the 
updates of design parameters. The proposed iterative algorithm is guaranteed to converge to a local optimal solution. With several design examples we have shown the capability of the proposed design methodology.

\section{References}

V.D. Blondel and J.N. Tsitsiklis, "A survey of computational complexity results in systems and control", Automatica, 36, pp. 1294-1274, 2000.

S.P. Boyd and C.H. Barratt, Linear Controller Design-Limits of Performance, Englewood Cliffs, NJ: Prentice Hall, 1991.

S. Boyd, L. El Ghaoui, E. Feron and V. Balakrishnan, Linear Matrix Inequalities in Systems and Control Theory, Philadelphia, PA: SIAM, 1994.

L.L. Chien, "Integrated plant and controller design using iterative redesign algorithms and linear matrix inequalities". Master's thesis, University of Houston (2000).

H. Doki, K. Hiramoto, I. Saito and T. Miyazaki, "A simultaneous optimal design for cantilevered pipes conveying fluid (Experimental verification for a combined pipe conveying fluid)", Trans. Japan Soc. Mech. Eng., C, 68, pp. 817-824, 2002 (in Japanese).

Y. Ebihara and T. Hagiwara, "New dilated LMI characterisations for continuous-time multiobjective controller synthesis", Automatica, 40, pp. 2003-2009, 2004.

P. Gahinet, A. Nemirovski, A.J. Laub and C. Chilali, LMI Control Toolbox for Use with MATLAB, Natick, MA: The Mathworks Inc., 1995.

J.C. Geromel, "Convex analysis and global optimisation of joint actuator location and control problems", IEEE Trans. Automat. Cont., 34, pp. 711-720, 1989.

K.M. Grigoriadis, G. Zhu and R.E. Skelton, "Optimal redesign of linear systems", Transactions of the ASME, J. Dyn. Sys., Meas. Cont., 118, pp. 598-605, 1996.

A. Hassibi, J. How and S. Boyd, "A path-following method for solving BMI problems in control", in Proc. Amer. Cont. Conf., pp. 1385-1389, 1999.

K. Hiramoto, H. Doki and G. Obinata, "Optimal sensor/actuator placement for active vibration control using explicit solutions of algebraic Riccati equation", J. Sound Vib., 299, pp. 1057-1075, 2000 .
K. Hiramoto and H. Doki, "Simultaneous optimal design of structural and control systems for cantilevered pipes conveying fluid", J. Sound Vib., 274, pp. 685-699, 2004.

T. Iwasaki, "The dual iteration for fixed-order control", IEEE Trans. Automat. Cont., 44, pp. 783-788, 1999.

T. Iwasaki, S. Hara and H. Yamauchi, "Structure/control design integration with finite frequency positive real property", in Proc Amer. Cont. Conf., pp. 549-553, 2000.

I. Kajiwara, K. Tsujioka and A. Nagamatsu, "Approach for simultaneous optimisation of a structure and control system", AIAA J., 32, pp. 866-873, 1994.

J. Lu and R.E. Skelton, "Integrating structure and control design to achieve mixed $H_{2} / H_{\infty}$ performance", Int. J. Cont., 73 , pp. 1449-1462, 2000.

C.S. Mehendale and K.M. Grigoriadis, "A homotopy method for decentralized control design", in Proc. Amer. Cont. Conf., pp. 5023-5027, 2003.

L. Meirovitch, Dynamics and Control of Structures, New York: John Wiley \& Sons, 1990, pp. 333-336.

M.C. de Oliveira and J.C. Geromel, "Linear output feedback design with joint selections of sensors and actuators", IEEE Trans. Automat. Cont., 45, pp. 2412-2419, 2000.

J. Onoda and R.T. Haftka, "An approach to structure/control simultaneous optimisation for large flexible spacecraft", $A I A A J$., 25, pp. 1133-1138, 1987.

J. Rakowska, R.T. Haftka and L.T. Watson, "Multi-objective controlstructure optimisation via homotopy methods", SIAM J. Optim., 3 pp. 654-667, 1993.

S.S. Rao, V.B. Venkayya and N.S. Khot, "Game theory approach for the integrated design of structure and controls", AIAA J., 26 , pp. 463-469, 1998.

C. Scherer, P. Gahinet and M. Chilali, "Multiobjective outputfeedback control via LMI optimisation", IEEE Trans. Automat. Cont., 42, pp. 896-911, 1997.

R.E. Skelton, T. Iwasaki and K. Grigoriadis, A Unified Algebraic Approach to Linear Control Design, London: Taylor \& Francis, 1998.

H. Tanaka and T. Sugie, "General framework and BMI formulae for simultaneous design of structure and control systems", Trans. Soc. Instrum. Cont. Eng., 34, pp. 27-33, 1998 (in Japanese).

G. Zhai, M. Ikeda and Y. Fujisaki, "Decentralized $\mathcal{H}_{\infty}$ controller design: a matrix inequality approach using a homotopy method", Automatica, 37, pp. 565-572, 2001. 
Copyright of International Journal of Control is the property of Taylor \& Francis Ltd and its content may not be copied or emailed to multiple sites or posted to a listserv without the copyright holder's express written permission. However, users may print, download, or email articles for individual use. 